\title{
Visualization of Neuromuscular Junctions over Periods of Several Months in Living Mice
}

\author{
Jeff W. Lichtman, Lorenzo Magrassi, and Dale Purves \\ Department of Anatomy and Neurobiology, Washington University School of Medicine, St. Louis, Missouri 63110
}

Identified neuromuscular junctions were followed in the sternomastoid muscle of living mice for several months by repeated staining with the fluorescent dye 4-(4-diethylaminostyryl)-N-methylpyridinium iodide (4-Di-2-ASP; Magrassi et al., 1987). Overall terminal growth occurred at many endplates; however, the branching pattern of presynaptic arbors was largely unchanged, even after several months. The absence of significant remodeling over time was not a result of dye-staining, since sprouting was readily induced at residual motor endings by partial denervation. We conclude that-apart from overall growth-most neuromuscular junctions in the adult mouse are stable over intervals that represent a significant fraction of the animal's lifetime.

The issue of whether synaptic terminals are static or dynamic is of considerable interest. In vertebrate muscle, the evidence on this point is conflicting. On one hand, postsynaptic specializations of the vertebrate neuromuscular junction (e.g., acetylcholine receptors, acetylcholinesterase) are remarkably stable in that they persist for long periods following denervation (Letinsky et al., 1976; Sanes et al., 1980; Steinbach, 1981). Moreover, the endplate, as defined by these specializations, is reinnervated quite precisely during axon regeneration in adult animals (Letinsky et al., 1976). On the other hand, morphological observations of fixed material have suggested that there is persistent remodeling of the neuromuscular junction (Barker and Ip, 1966; Tuffery, 1971; Kemplay and Stolkin, 1980; see also TownesAnderson and Raviola, 1978). More detailed evidence for continual morphological change at neuromuscular junctions has been provided by descriptions of presynaptic elements unopposed by postsynaptic specializations, and of postsynaptic structures lacking a corresponding nerve terminal (Wernig et al., 1980; Cardasis and Padykula, 1981; Haimann et al., 1981; Anzil et al., 1984; Wernig et al., 1984). These arrangements have been interpreted, respectively, as sprouts that have not yet induced subsynaptic specializations and recently vacated synaptic sites. Finally, comparison of old and young neuromuscular junctions indicates that endplates become more complex as a function of

\footnotetext{
Received June 10, 1986; revised Sept. 16, 1986; accepted Sept. 25, 1986.

This work was supported by NIH Grants NS 11699, 18629, and 20364; a Javits Center grant; grants from the Muscular Dystrophy Association; and a McKnight Research Development Award to J.W.L. We are grateful to Pat Newton for skillful assistance, to C. J. Forehand, R. D. Hadley, W. D. Snider, and J. Voyvodic for useful comments on the manuscript, and to P. Bridgman for the loan of equipment.

Correspondence should be addressed to Dr. Dale Purves, Department of Anatomy and Neurobiology, Washington University School of Medicine, 660 South Euclid Avenue, St. Louis, MO 63110.
}

Copyright (C) 1987 Society for Neuroscience $0270-6474 / 87 / 041215-08 \$ 02.00 / 0$ normal aging (see, for example, Fahim et al., 1983; Hopkins et al., 1985).

In the preceding paper (Magrassi et al., 1987), we evaluated a series of fluorescent dyes that stain neuromuscular junctions

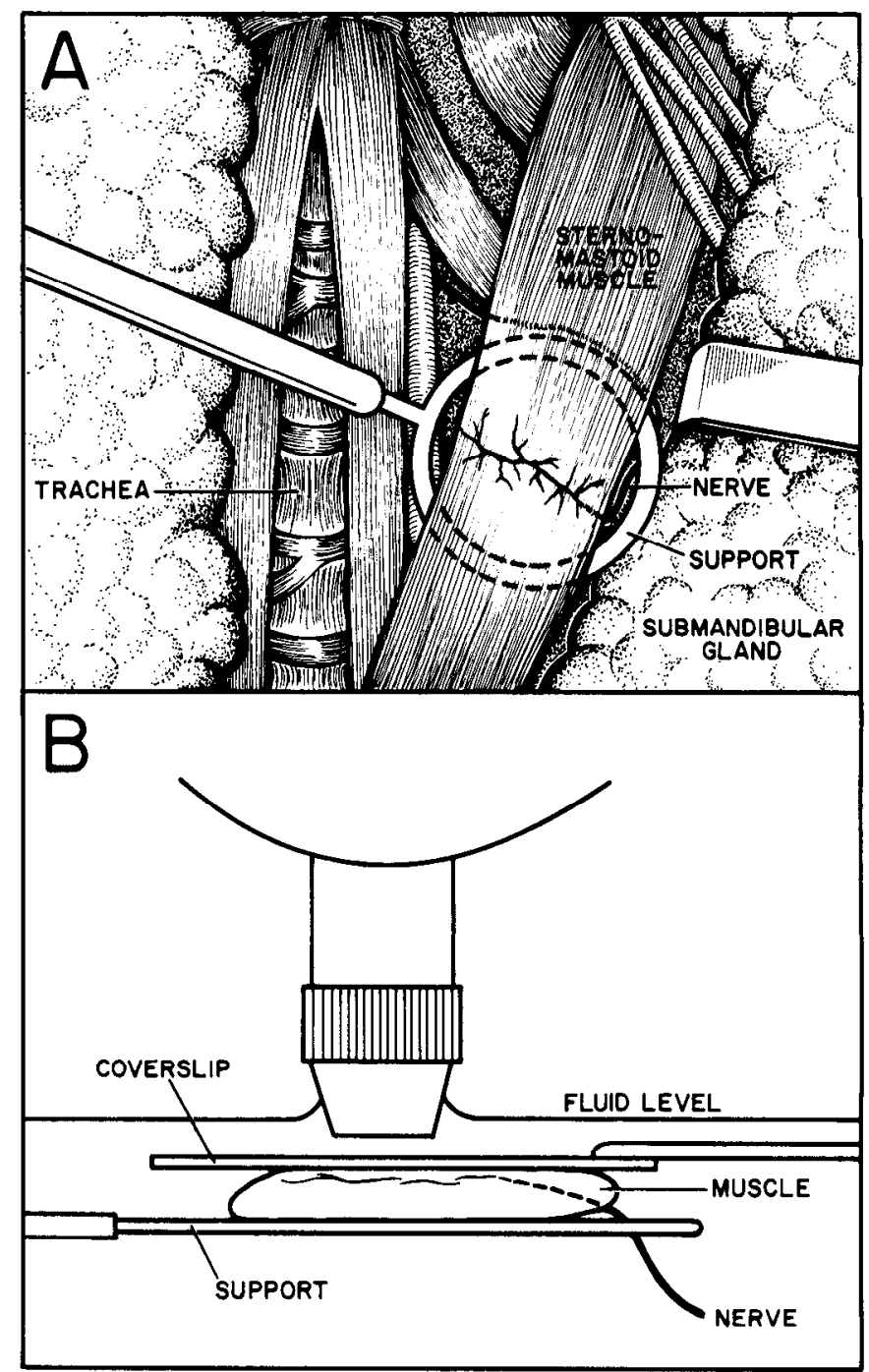

Figure 1. Method of viewing motor nerve terminals on the surface of the sternomastoid muscle in a living mouse. $A$, Diagram of the left sternomastoid muscle, its nerve supply, and the position of the endplate band on its ventral surface. The muscle belly is supported by a stainless steel ring after retracting the submandibular gland. $B$, Side view showing muscle supported by the ring and lightly compressed by a coverslip to flatten and stabilize the muscle surface. 

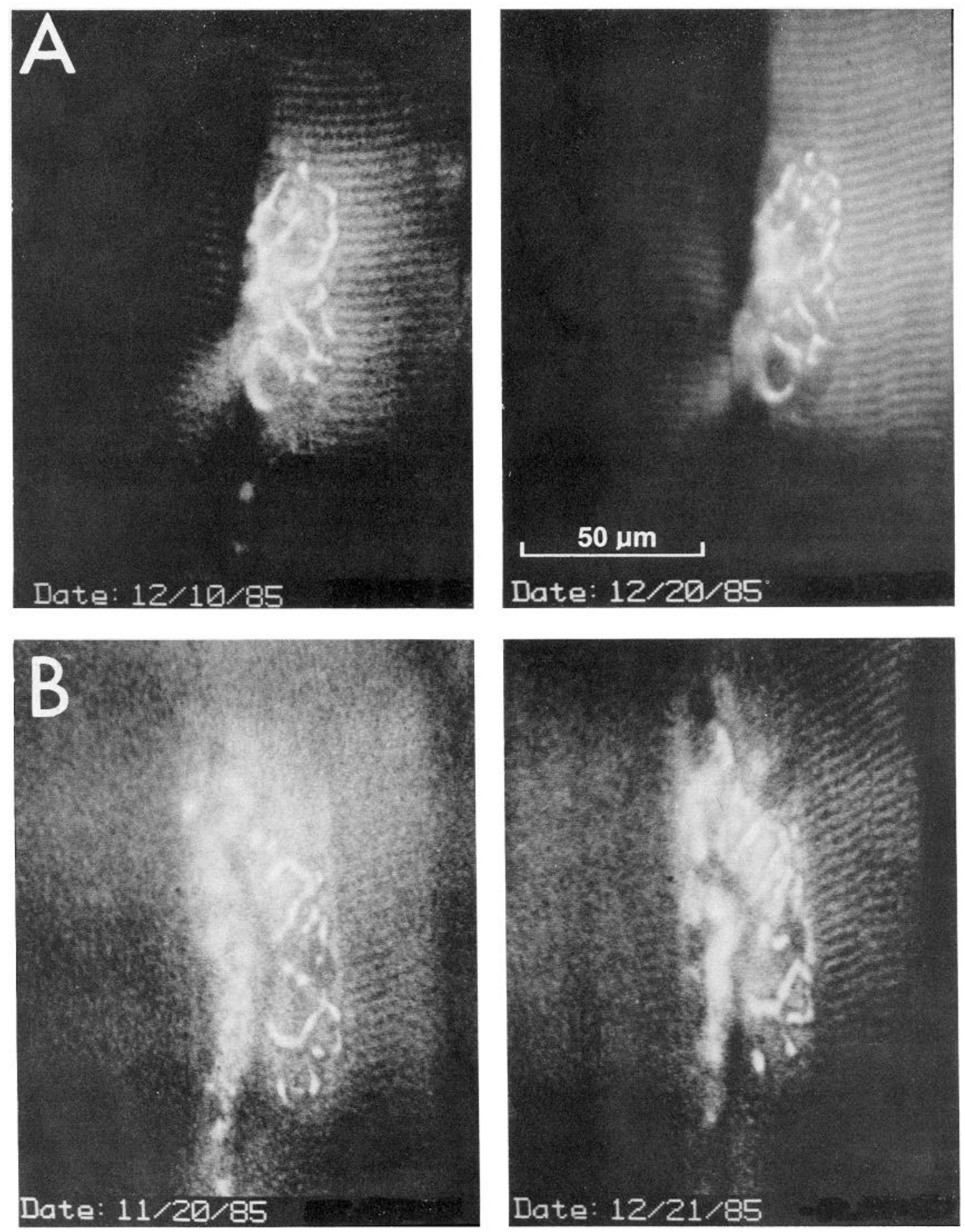

Figure 2. Nerve terminals on mouse sternomastoid muscle fibers viewed over relatively short intervals. $A$, Terminal arbor visualized over an interval of $10 \mathrm{~d}$. $B$, Terminal arbor visualized over an interval of $30 \mathrm{~d}$. No obvious changes were noted over periods of up to $30 \mathrm{~d}$.

in living animals. In the present report we have used the best of these reagents-4-(4-diethylaminostyryl)- $N$-methylpyridinium iodide (4-Di-2-ASP) - to monitor directly the morphology of single, identified neuromuscular junctions over intervals of up to 6 months. These observations on living nerve terminals in the mouse indicate that there is considerable growth of most terminal arbors. There is, however, little or no rearrangement of motor endings under normal circumstances at this level of the nervous system.
Some of these results have been briefly reported (Lichtman et al., 1986).

\section{Materials and Methods}

Young adult mice of either sex (CF1 strain; weight, 22-32 gm) were anesthetized with chloral hydrate $(0.6 \mathrm{gm} / \mathrm{kg})$. The animals were placed on the stage of a compound microscope, the upper portion of which could be rotated out of the way so that a dissecting microscope could also be placed over the animal (see also Purves et al., 1986). The neck was then shaved, the skin washed with antiseptic solution, and a midline 
incision made from the apex of the mandible to the sternal notch. An endotracheal tube was inserted to enable control of the animal's breathing by a respirator throughout the procedure. The ventral surface of the left sternomastoid muscle was exposed and superfused with sterile saline (lactated Ringer's solution; Travenol) (Fig. 1A). The perfusion system was attached to a 3-way stopcock so that the dye solution could be introduced after passage through a $0.2-\mu \mathrm{m}$-pore filter. The muscle was stained with a $10 \mu \mathrm{M}$ solution of 4-Di-2-ASP (Molecular Probes, Inc. Eugene, OR) in Ringer's fluid (see Magrassi et al., 1987). At the end of the 3 min staining period, the excess dye was flushed from the wound with lactated Ringer's for a further minute. The muscle was then gently lifted onto a wire loop to raise it above the surface of the pool; the endplate zone, which lies in the midregion of the muscle (see Fig. 1A), was flattened slightly with a $12 \mathrm{~mm}$ circular coverslip cemented to a light metal spring (Fig. 1B). This arrangement served to improve the optics, stabilize the muscle, and bring most of the endplate region into the same focal plane.

To minimize illumination of the stained motor endings, we visualized the endplate band with a silicon intensified tube (SIT) camera (GE 4Te56 or Dage 66), a variable-intensity light source (100 W Osram Xenophot bulb), and conventional epifluorescence optics (Leitz H2 cube; 390-490 $\mathrm{nm}$ excitation, $515 \mathrm{~nm}$ suppression). After recording the general arrangement of the endplate region at low power, we chose several endplates $(1-5)$ for detailed study. The terminal arbors selected were viewed on a video monitor using 25,50 , or $100 \times$ water-immersion objectives (numerical apertures, $0.60,1.00$, or 1.20 , respectively). Video images were digitized, averaged $(\times 8)$, and stored on a disc. For this purpose, an image processor (TRAPIX; Recognition Concepts) was used in conjunction with a Micro-Vax II computer (DEC) and the IMAGR program, written by J. Voyvodic (1986). In some experiments, a Hughes image processor was used (model 794). Once images of the selected endplates had been obtained, the wound was closed and the animals allowed to recover. The stored images were then recalled and photographed on Polaroid film after digital contrast enhancement.

After an interval of 5-180 d, the entire procedure was repeated exactly. The endplates stained at the first operation could usually be found promptly by scanning the muscle surface at low power while referring to the pictures taken at the initial operation. Additional photographs of the identified motor terminals were then acquired in the same manner as at the initial operation.

Measurements of endplate areas and terminal lengths were made from photocopies of stained terminals with a digitizing pad (Summagraphics) attached to the computer.

\section{Results}

\section{Motor nerve terminals visualized over intervals of up to one month}

At each of 50 neuromuscular junctions visualized within a period of a month or less ( 23 animals), the configuration of motor nerve terminals appeared to be unchanged (Fig. 2). Minor differences could be attributed to slight changes in orientation or in the degree of compression exerted by the coverslip. As a general rule, instances in which there was some question of an altered configuration occurred when the terminal was poorly seen at one viewing or the other (for example, when the endplate was slightly out of focus or located on the edge of a fiber). In all cases in which homologous branches could be seen clearly at the beginning and the end of the experiment, the configuration of the terminals failed to show any obvious change.

\section{Ability of stained motor nerve terminals to sprout}

Because most mouse neuromuscular junctions appeared stable over intervals of up to a month, we felt it important to assure ourselves that motor nerve terminals that had been stained with 4-Di-2-ASP were indeed capable of change. Thus, in 2 additional animals we produced partial denervation by lightly pinching the nerve to the sternomastoid muscle (see Fig. 1). When the muscle was viewed again 1-2 weeks after this procedure, numerous endplates showed signs of sprouting (Fig. 3). Staining

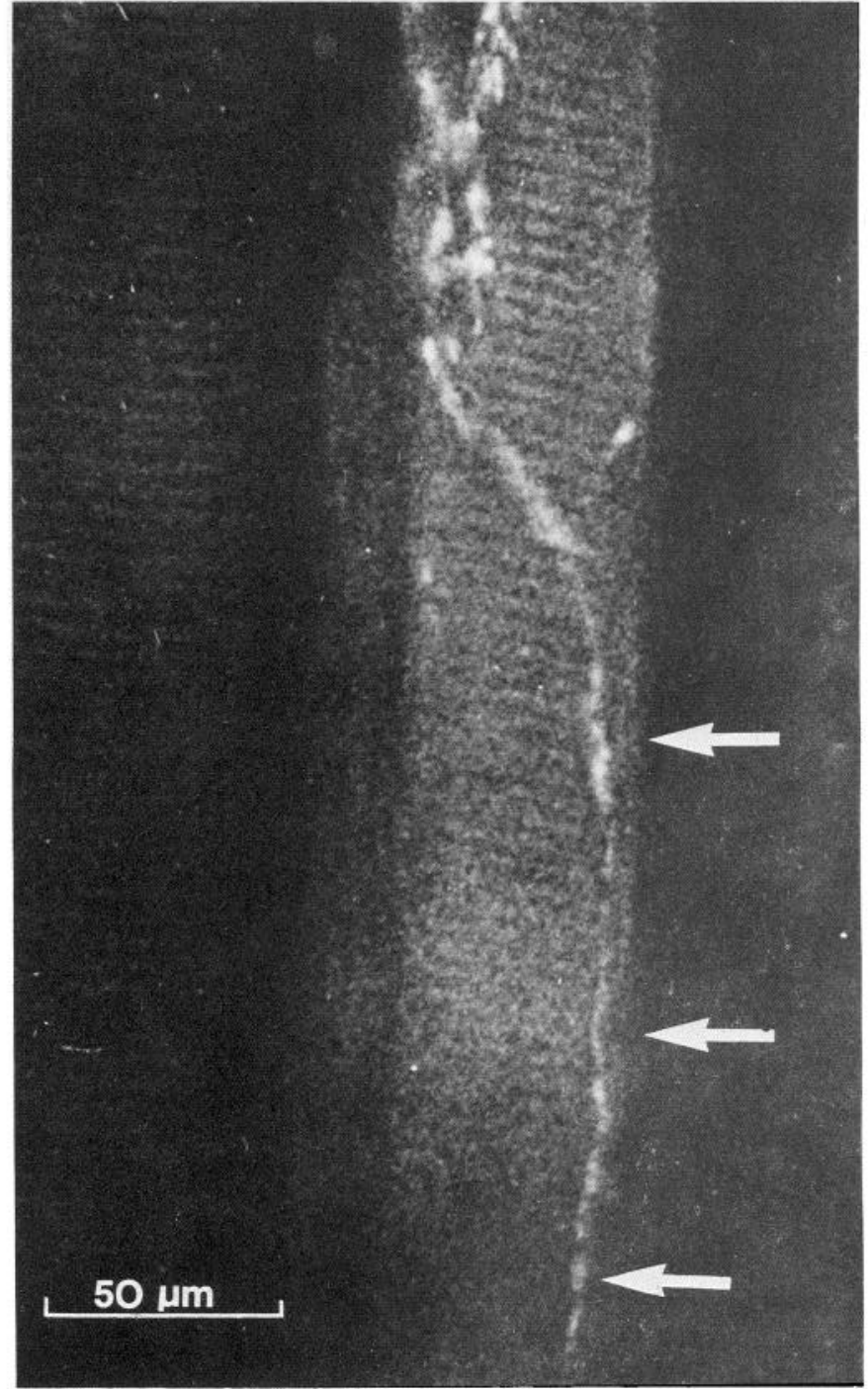

Figure 3. Sprout (arrows) arising from stained nerve terminal after partial crush of the sternomastoid motor nerve branch at the time of initial staining 1 week earlier. Many residual terminals showed evidence of sprouting after partial denervation. No effort was made to study identified terminals in this circumstance because a large and variable number of terminals disappeared after the denervation procedure.

with 4-Di-2-ASP, then, does not impede the ability of motor terminals to extend new processes; by the same token, the staining procedure we used is quite capable of revealing newly formed sprouts.

\section{Motor nerve terminals visualized at longer intervals}

It seemed quite possible that an interval of a month or less might be insufficient for demonstrating slow changes in the configuration of motor nerve terminals. We therefore followed a comparable number of animals over periods of 3-6 months.

At 41 endplates that were reidentified at the end of 3-4 months (13 animals), the configurations of the terminal branches at the beginning and end of this period were similar (Fig. 4). However, in many (but not all) cases, the overall size of the terminal arbor had increased substantially (Figs. 4, 5; see also Fig. 6). Because the complexity of the terminal arborizations did not change appreciably over this interval, the increased area occupied by 

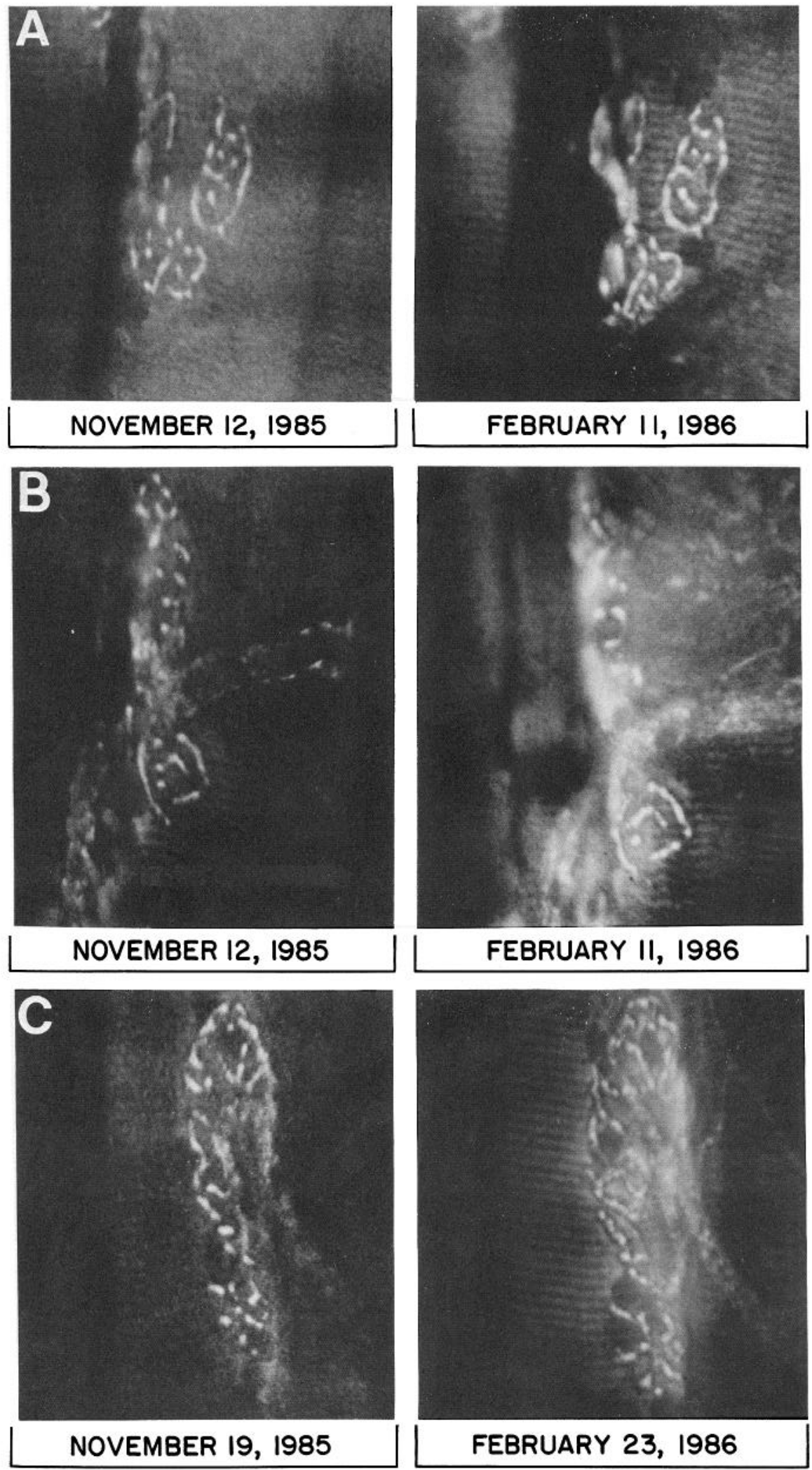

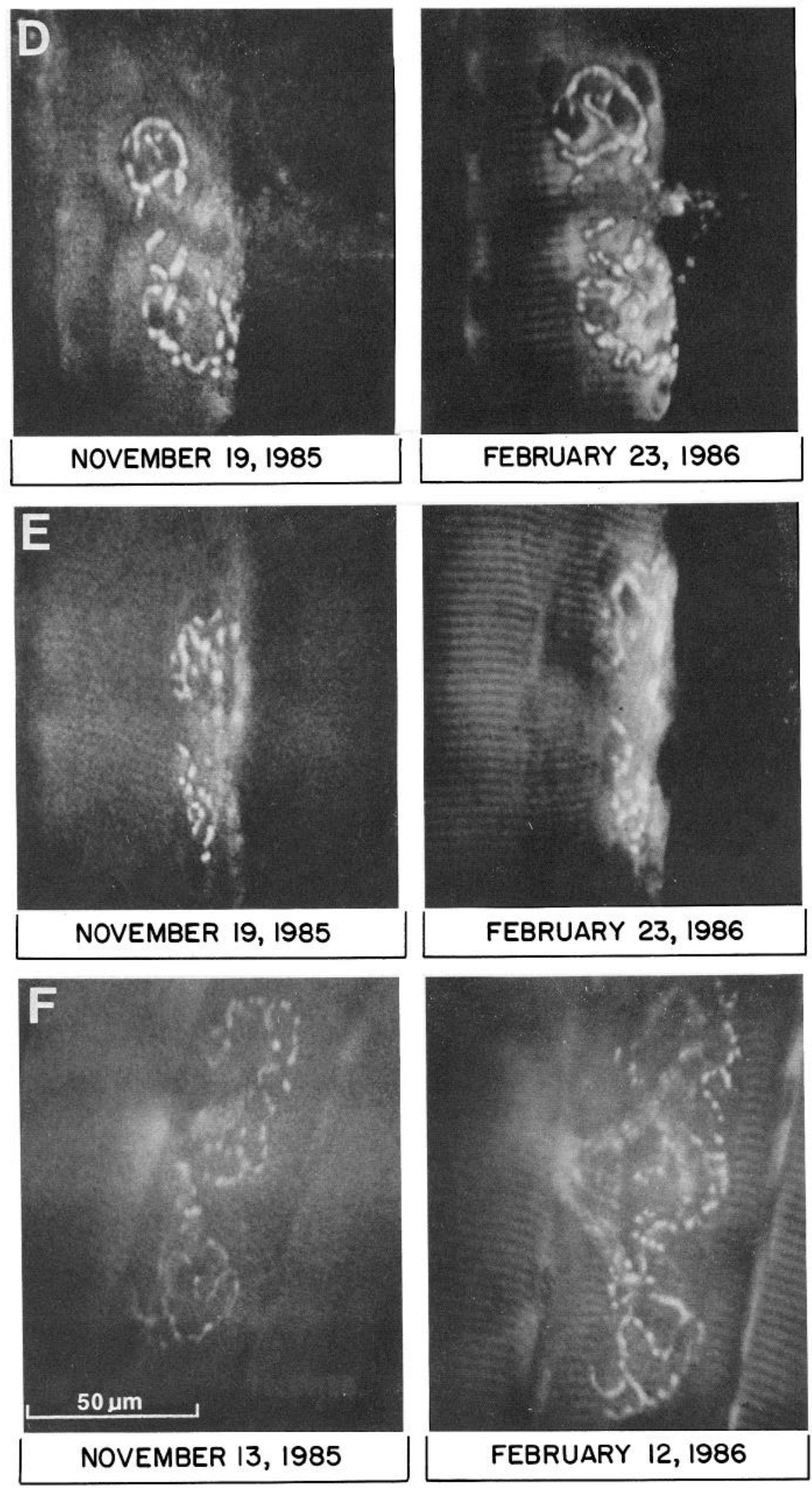

Figure 4. Nerve terminals viewed over an interval of 3 months. $A-F$, Representative examples of endplates studied over this interval. As in Figure 2, the left panel of each pair shows the terminal arbor at the beginning of the interval and the right panel the terminal at the end of the experiment. Particular elements in the terminal arbor were easily reidentified and continued to bear the same relationship to one another. The overall area occupied by the terminal branches, however, increased (see Fig. 5). 


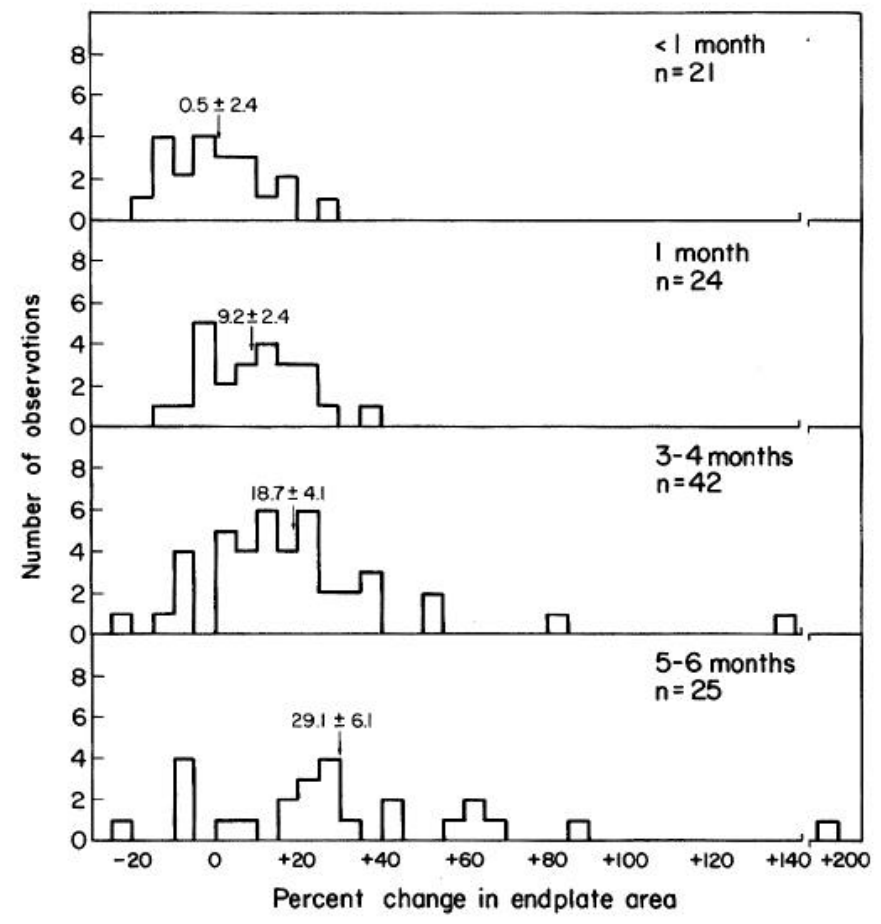

Figure 5. Distribution of the changes in the area occupied by the terminal branches at 112 endplates as a function of the interval between observations. Area measurements were made by tracing the perimeter of the region occupied by terminal branches (see, for example, Figs. 2 4); change is expressed as percent increase (or decrease) over each interval. Little or no change was observed over intervals of up to a month however, most endplates had enlarged substantially by 3-4 months. The average weight increase of the animals was $6 \%$ for intervals of less than 1 month, $19 \%$ for intervals of 1 month, 29\% for intervals of 3-4 months and $32 \%$ for intervals of 5-6 months. Arrows indicate the means of each distribution.

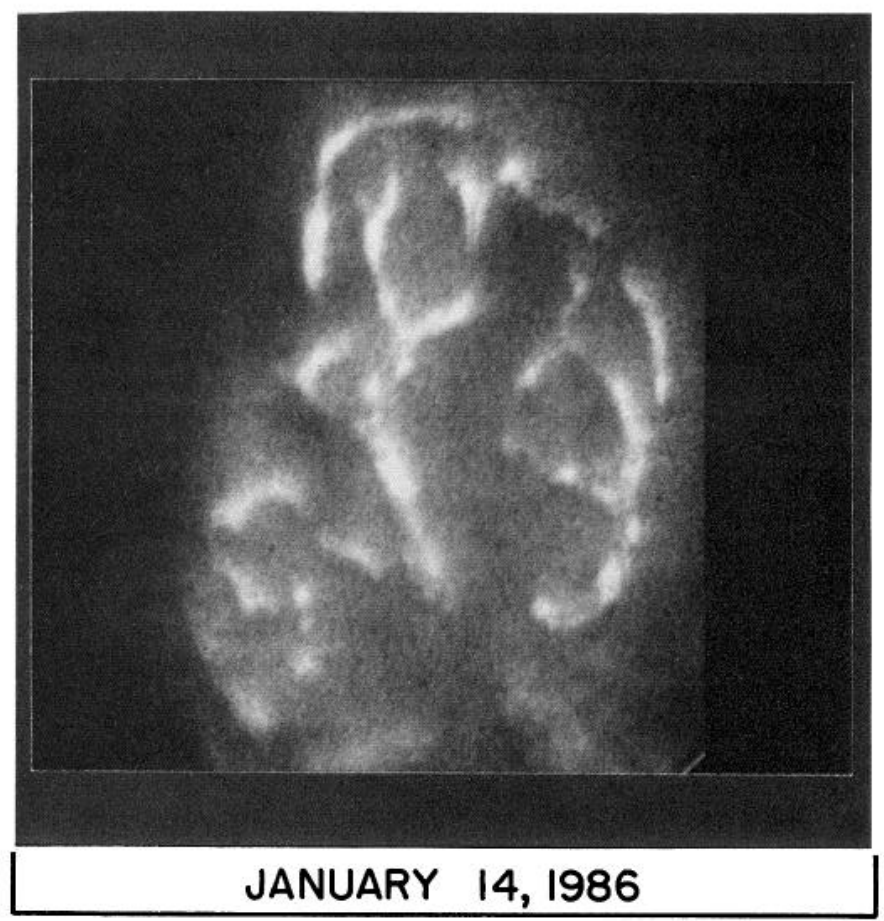

the terminals implies proportional growth of existing branches. To ensure that we were not missing smaller branches not well resolved by the $25 \times$ objective we routinely used, we also examined some terminals with $50 \times$ or $100 \times$ objectives with higher numerical apertures. The general appearance of the terminals was similar, although minor differences were more apparent at higher magnification (Fig. 6).

The longest interval over which terminals were followed was 5-6 months. Even at this interval, the major change that we observed at most of the 27 endplates studied (10 animals) was overall enlargement of the terminal arborization (Fig. 7). As at the 3-4 month interval, this increase in the area occupied by the terminal branches was apparently achieved by a gradual growth of individual presynaptic elements that maintained their general relationship. At only 1 endplate (Fig. 8) was there a clear indication that a substantial terminal branch had been added (or withdrawn).

\section{Discussion}

The results reported here show that most motor nerve terminals on skeletal muscle fibers in the adult mouse gradually enlarge (presumably as the animals themselves grow; see Hopkins et al., 1985), but are otherwise stable over periods representing a substantial fraction of the animal's lifetime (up to $20 \%$ in the mouse).

Some degree of sprouting does occur normally at neuromuscular junctions in mammals and other animals. These sproutsdefined as presynaptic terminal processes that extend away from the endplate for a substantial distance (see Fig. 3)-are sometimes seen on muscle fibers using any of several techniques (including the ones employed here). Thus we occasionally observed an obvious terminal sprout at normal junctions in the mouse sternomastoid, although we did not happen to see any

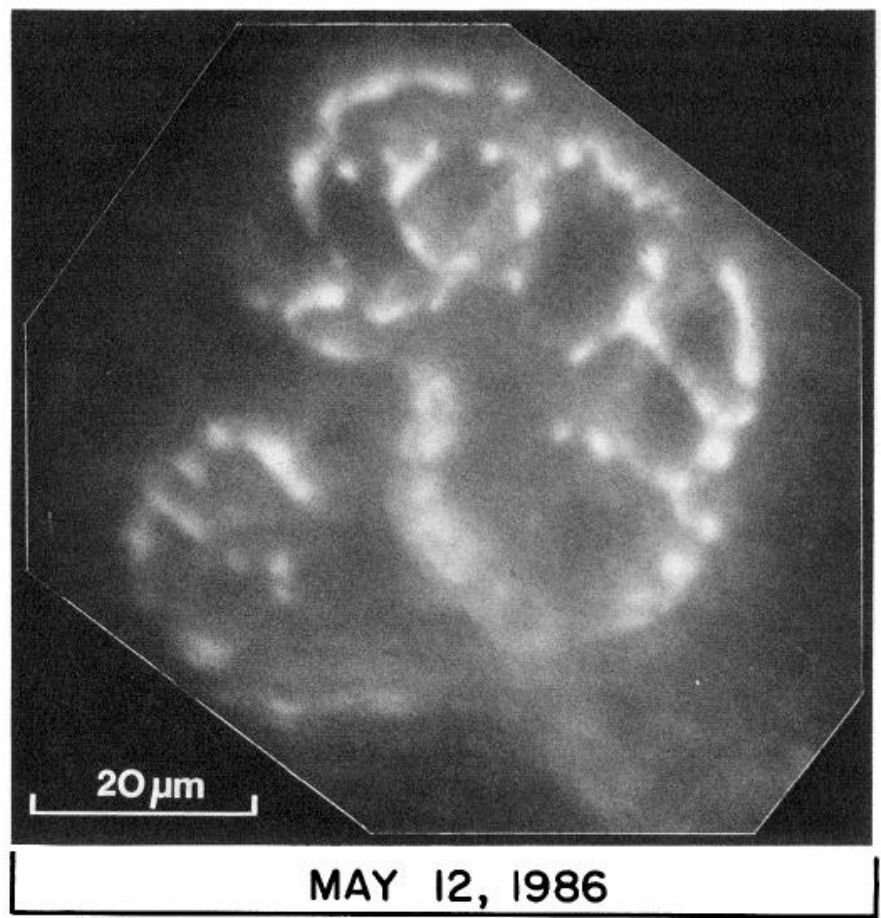

Figure 6. Nerve terminal viewed at higher power using a $100 \times$ water-immersion objective (primary magnification, $1250 \times ;$ numerical aperture $=$ 1.2) initially (left) and after 4 months (right). Apart from overall growth, the changes in terminal configuration over this interval are small. The total length of the terminal branches at this endplate increased from 387 to $522 \mu \mathrm{m}$. Much of the enlargement was due to lengthening of existing branches. There were, however, some slight changes (e.g., the bifurcation at the left end of the top branch). In virtually all cases, such refinements were minor at the intervals studied (up to 6 months). 

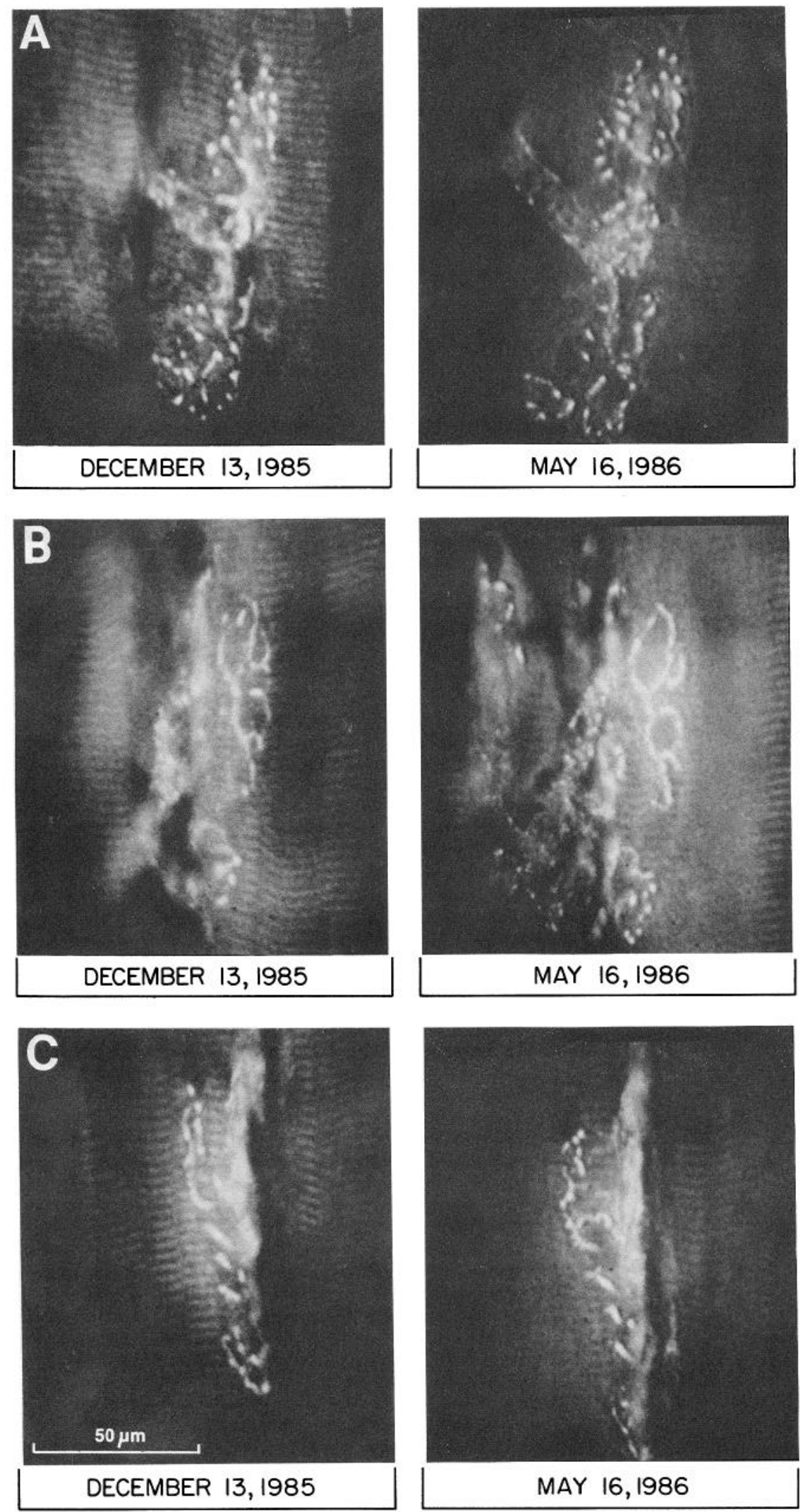

Figure 7. $A-C$, Nerve terminals viewed initially $(l e f t)$ and 6 months later (right). The major change is again an increased area occupied by the terminal arbor. Minor changes in terminal configuration, however, are somewhat more apparent after 6 months than at shorter intervals (cf. Figs. 2, 4, 6). during the initial viewing at the junctions chosen for detailed study. The fact that only one of the 118 endplates that we followed over periods of up to 6 months showed a major change in terminal configuration argues for the rarity of terminal sprouting under normal conditions. Clearly, the sprouting that does occur normally is not a process that significantly influences the morphology of most motor terminals. The minor changes that we did observe in the configuration of some endplates after several months presumably arose from a more subtle process.

It is interesting to compare the present results on the stability 

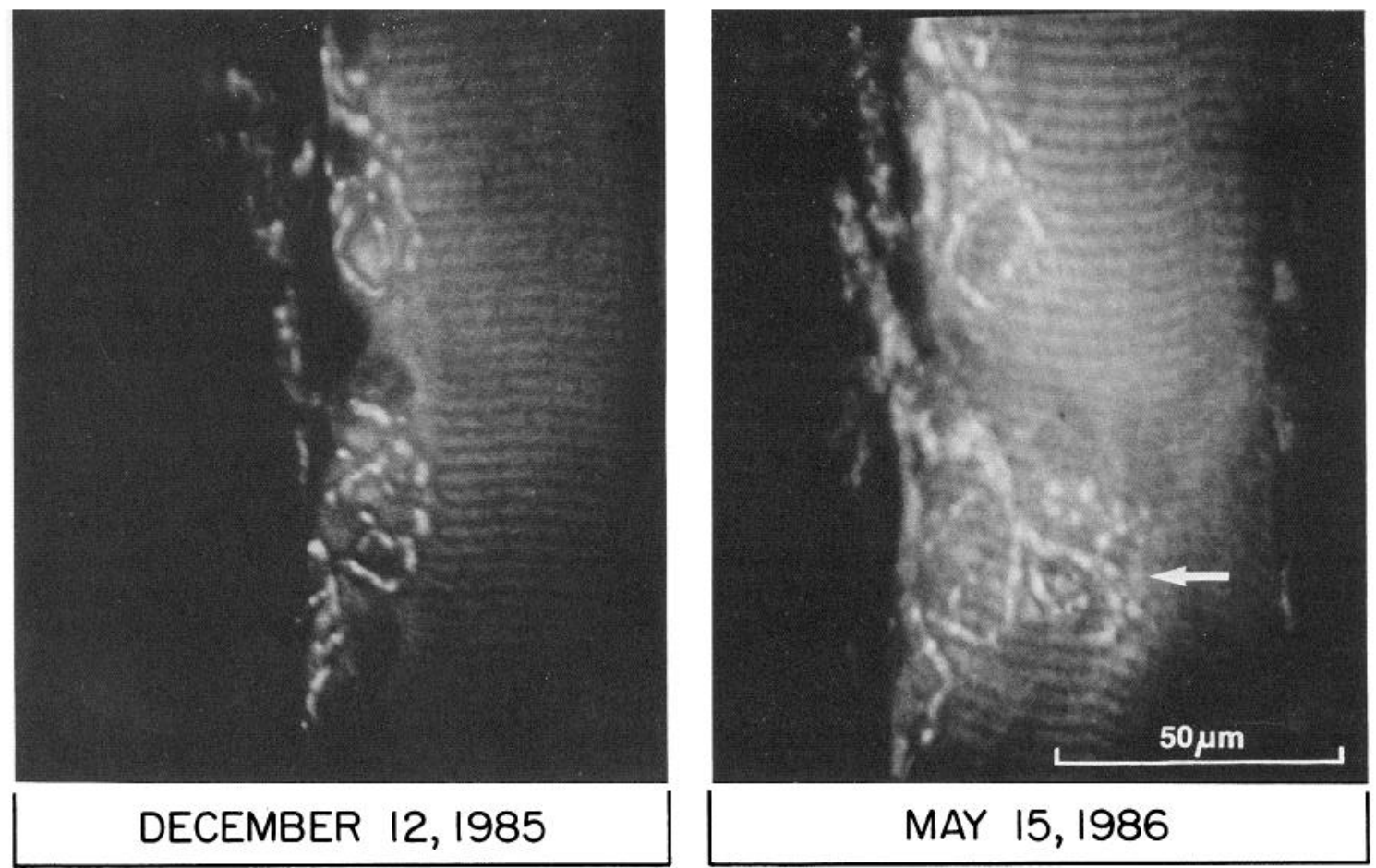

Figure 8. A motor terminal that sprouted to form a new terminal complex sometime between the initial observation and the second observation 6 months later. Arrow indicates the portion of the terminal that was added. This was the only example in the 118 terminal arbors studied in which such a change occurred.

of motor nerve terminals in living animals with those of similar studies of the dendritic processes of another class of motor neurons, autonomic ganglion cells (Purves and Hadley, 1985; Purves et al., 1986). Like axon terminals, the dendritic arbors of superior cervical ganglion cells show considerable net growth over periods of several months. However, dendritic branches appear to be more dynamic than axon terminals in that the configuration of dendritic arbors changes substantially over intervals similar to the ones we have examined here. This occurs through the extension of some branches and the retraction of others. It will be of interest to examine these apparent differences between the terminals of axons and dendrites in a variety of preparations in order to see what general rules may be discerned.

\section{References}

Anzil, A. P., A. Beiser, and A. Wernig (1984) Light and electron microscopic identification of nerve terminal sprouting and retraction in normal adult frog muscle. J. Physiol. (Lond.) 350:393-399.

Barker, D., and M. C. Ip (1966) Sprouting and degeneration of mammalian motor axons in normal and deafferentated skeletal muscle. Proc. R. Soc. Lond. [Biol.] 163: 538-554.

Cardasis, C. A., and H. A. Padykula (1981) Ultrastructural evidence indicating reorganization at the neuromuscular junction in the normal rat soleus muscle. Anat. Rec. 200: 41-59.

Fahim, M. A., J. A. Holley, and N. Robbins (1983) Scanning and light microscopic study of age changes at a neuromuscular junction in the mouse. J. Neurocytol, 12: 13-25.

Haimann, C., A. Mallart, J. Tomás i Ferré, and N. F. Zilber-Gachelin (1981) Patterns of motor innervation in the pectoral muscle of adult Xenopus laevis: Evidence for possible synaptic remodelling. J. Physiol. (Lond.) 310: 241-256.

Hopkins, W. G., M. C. Brown, and R. J. Keynes (1985) Postnatal growth of motor nerve terminals in muscles of the mouse. J. Neurocytol. 14: 525-540.

Kemplay, S., and C. Stolkin (1980) Endplate classification and spontaneous sprouting in the sternocostalis muscle of the rat: A new whole mount preparation. Cell Tissue Res. 212: 333-339.
Letinsky, M. S., K. H. Fischbeck, and U. J. McMahan (1976) Precision of reinnervation of original postsynaptic sites in frog muscle after a nerve crush. J. Neurocytol. 5: 691-718.

Lichtman, J. W., L. Magrassi, and D. Purves (1986) Visualization of motor nerve terminals in living mice. Soc. Neurosci. Abstr. 12: 390.

Magrassi, L., D. Purves, and J. W. Lichtman (1987) Fluorescent probes that stain living nerve terminals. J. Neurosci. 7: 1207-1214.

Purves, D., and R. D. Hadley (1985) Changes in the dendritic branching of adult mammalian neurones revealed by repeated imaging in situ. Nature 315: 404-406.

Purves, D., R. D. Hadley, and J. Voyvodic (1986) Dynamic changes in the dendritic geometry of individual neurons visualized over periods of up to three months in the superior cervical ganglion of living mice. J. Neurosci. 6: 1051-1060.

Sanes, J. R., L. M. Marshall, and U. J. McMahan (1980) Reinnervation of skeletal muscle: Restoration of the normal synaptic pattern. In Nerve Repair: Its Clinical and Experimental Basis, D. L. Jewett and H. R. McCarroll, eds., pp. 130-138, Mosby, St. Louis, MO.

Steinbach, J. H. (1981) Neuromuscular junctions and $\alpha$-bungarotoxinbinding sites in denervated and contralateral cat skeletal muscles. J. Physiol. (Lond.) 313: 513-528.

Townes-Anderson, E., and G. Raviola (1978) Degeneration and regeneration of autonomic nerve endings in the anterior part of the rhesus monkey ciliary muscle. J. Neurocytol. 7: 583-600.

Tuffery, A. R. (1971) Growth and degeneration of motor end-plates in normal cat hind limb muscles. J. Anat. 110: 221-247.

Voyvodic, J. T. (1986) A general purpose image processing language (IMAGR) facilitates visualizing neuronal structures in fixed tissues and in vivo. Soc. Neurosci. Abstr. 12: 390.

Wernig, A., M. Pécot-Dechavassine, and H. Stöver (1980) Sprouting and regression of the nerve at the frog neuromuscular junction in normal conditions and after prolonged paralysis with curare. J. Neurocytol. 9: 277-303.

Wernig, A., J. J. Carmody, A. P. Anzil, E. Hansert, M. Marciniak, and H. Zucker (1984) Persistence of nerve sprouting with features of synapse remodelling in soleus muscles of adult mice. Neuroscience 11: 241-253. 